\title{
Propiedades psicométricas del inventario psicológico de ejecución deportiva (IPED) en una muestra de nadadores de Chile
}

\author{
Psychometric properties of the psychological inventory of sports execution (IPED) \\ in a swimmer sample of Chile \\ *Carlos Véliz Véliz, **Fernando Maureira Cid, ***José Manuel Laurido Huepe, \\ $* * *$ Nakia Milko Carmi Manacilla, ***Rodrigo Andrés Cortés Rojas
}

Véliz, C., Maureira, F., Laurido, J.M., Carmi, N. \& Cortés, R. (2018). Propiedades psicométricas del inventario psicológico de ejecución deportiva (IPED) en una muestra de nadadores de Chile. Revista Ciencias de la Actividad Física UCM, $\mathrm{N}^{\circ}$ 19(2) julio-diciembre, 1-10. DOI: http://doi.org/10.29035/rcaf.19.2.3

\section{RESUMEN}

El objetivo del presente estudio fue conocer las propiedades psicométricas del Inventario Psicológico de Ejecución Deportiva (IPED) en nadadores federados de Chile. Para ello se evaluaron 99 sujetos de los cuales 41 son damas $(41,4 \%)$ y 58 son varones $(58,6 \%)$. Los resultados muestran que las sub-escalas de autoconfianza, control de afrontamiento negativo, control visuo-imaginativo y nivel emocional estaban compuestas por un solo factor que explicaba entre el $37 \%$ y $42 \%$ de la varianza, en tanto, las sub-escalas de control atencional, control de afrontamiento positivo y control actitudinal estaban compuestas por dos factores que explicaban entre el $57 \%$ y $67 \%$ de la varianza total. La consistencia interna de cada sub-escala fue aceptable. Los resultados muestran que este inventario es confiable y valido en la muestra utilizada. Son necesarias futuras investigaciones en una muestra más amplia que abarque nadadores de todo el país.

\section{PALABRAS CLAVE}

Factores psicológicos, desempeño deportivo, validez, confiabilidad, nadadores.

\begin{abstract}
The aim of the present study was to understand the psychometric properties of the Psychological Inventory of Sports Execution (IPED) in national swimmers of Chile. There were 99 subjects evaluated, of which 41 were ladies $(41,4 \%)$ and 58 were males $(58,6 \%)$. The results show that the sub-scales of self-confidence, negative confrontation control, visual-imaginative control and emotional level were composed by an alone factor that was explaining between $37 \%$ and $42 \%$ of the variance, while, the sub-scales of attentional control, positive confrontation control and attitudinal control were composed by them by two factors they were explaining between $57 \%$ and $67 \%$ of the total variance. The internal consistency of every sub-scale was acceptable. The results show that this inventory is reliable and valid in the used sample. Future investigations are necessary with a bigger sample that includes swimmers from the entire country.
\end{abstract}

\section{Key words}

Psychological factors, sports performance, validity, reliability, swimmers.

* Gimnasio Pulse, Estadio Mayor. Santiago de Chile.

** Escuela de Educación en Ciencias del Movimiento y Deportes, Universidad Católica Silva Henríquez. Santiago de Chile.

*** Universidad Mayor. Santiago de Chile. 


\section{INTRODUCCIÓN}

El principal objetivo en el deporte de alta competencia es que los atletas rindan al máximo de sus posibilidades y logren buenos resultados (Gómez, Granero \& Isorna, 2013). García, Rodríguez, Andrade \& Arce (2006) afirman que en el deporte de alto rendimiento existen variables de naturaleza psicológicas que son fundamentales para el éxito deportivo, algunas de ellas son la ansiedad, autocontrol, estados de ánimo, estrés, motivación y las habilidades interpersonales. Según Orlick \& Partington (1988) las incorporaciones de elementos psicológicos en el deporte han generado gran efectividad en el desarrollo del potencial de los atletas. Las investigaciones han mostrado, por ejemplo, que los deportistas con más fortaleza mental poseen más confianza en sus habilidades, mayor capacidad para mantenerse focalizado durante sus rutinas y también alta motivación, regulando con menos complejidad sus estados emocionales (Crust, 2009, Gómez, Granero \& Isorna, 2013, Gucciardi, 2012, Jones, Hanton, y Connaughton, 2007, López, García, Berengüi, Martínez, Baños, Torres, et al., 2011).

Uno de los instrumentos más utilizados para estudiar factores psicológicos que influyen en el desempeño deportivo ha sido el Inventario Psicológico de Ejecución Deportiva (IPED) de Hernández (2006). Este cuestionario fue creado a partir del Psychological Performance Inventory (PPI) de Loehr $(1982,1986,1990)$.

Izquierdo, Ruiz, Navarro, Mendoza, Rodríguez \& Brito (2006), aplicaron el IPED a 196 jugadores de la Federación Interinsular de Fútbol de la Palmas (España) para analizar la autoconfianza, control de afrontamiento negativo, control atencional, control visual e imaginativo, nivel motivacional, control de afrontamiento positivo y el control actitudinal. Los investigadores encontraron que los jugadores de categoría nacional eran los que tenían más confianza en sí mismos y, por el contrario, los jugadores de categorías menores poseían niveles más bajos de confianza, esto explicado por su poca experiencia en competencias y corta edad. Por otro lado, los autores mostraron que los jugadores de base tenían problemas para afrontar situaciones adversas y los jugadores motivados por la eficacia deportiva tenían mejores resultados que los que se preocupaban solo por el éxito.

En otro estudio, Álvarez, Estevan, Falcó, Hernández-Mendo \& Castillo (2014) estudiaron a 89 taekwondistas universitarios de competición (50 hombres y 39 mujeres) con edades entre 18 y 34 años. Los investigadores encontraron que todos los deportistas presentaban valores aceptables en sus habilidades psicológicas. Además, mostraban que los deportistas que seguían un programa de entrenamiento psicológico reportaban mayor control visuo-imaginativo así como un elevado afrontamiento positivo y más control actitudinal, que aquellos deportistas que no lo hacían.

Reyes, Raimundi \& Gómez (2012) aplicaron un programa de entrenamiento psicológico a 15 jugadoras de voleibol con edades entre 13 y 16 años. La intervención duro cuatro meses con 29 sesiones de 45 minutos cada uno. Los resultados arrojaron diferencias significativas antes y después de la intervención en autoconfianza $(\mathrm{p}=0,020)$, energía negativa ( $\mathrm{p}$ $=0,025)$ y control de la atención $(\mathrm{p}=0,002)$.

En relación con las características psicométricas del IPED, Raimundi, Reigal \& Mendo (2016) adaptaron y validaron el cuestionario en deportistas argentinos, encontrando una fiabilidad de 0,70 y 0,80 para todos las sub-escalas. Hernández-Mendo, Morales-Sánchez \& Peñalver (2014) aplicaron el IPED a 3434 participantes de diferentes disciplinas (natación, waterpolo, triatlhon, ciclismo, fútbol, baloncesto, balonmano, volleyball, skate y atletismo, entre otros) observando niveles de fiabilidad óptimos en 4 de las 7 escalas (control de afrontamiento positivo, control afrontamiento negativo, nivel motivacional y control atencional); finalmente, los investigadores encontraron coincidencia con la versión original de inventario, demostrando que es una herramienta óptima para evaluar variables psicológicas en el rendimiento deportivo. Por último, Izquierdo, Ruiz, Navarro, Mendoza, Rodríguez \& Brito 
(2006) aplicaron el IPED a 196 jugadores de la Federación Interinsular de Fútbol de la Palmas, encontrando valores de confiabilidad de $\alpha=0,802$.

En base a los antecedentes mencionados es que surge el objetivo de la presente investigación: conocer las propiedades psicométricas del Inventario Psicológico de Ejecución Deportiva en nadadores federados de Chile.

\section{METODOLOGÍA}

Tipo y diseño: la presente investigación es tipo descriptiva y de diseño no experimental.

Muestra: de tipo no probabilística. Estuvo constituida por 99 deportistas de natación de Chile. Los criterios de inclusión fueron la práctica de natación por al menos 1 año y haber participado en alguna competencia a nivel nacional o internacional en el último año. La edad de la muestra varía entre 13 y 21 años con una media de 15,2 años (d.e. $=1,81)$. Del total de deportistas, 41 son damas $(41,4 \%)$ y 58 son varones $(58,6 \%)$. El 53,5\% de los nadadores evaluados no han participado nunca en competencias como seleccionados nacionales, en tanto el $46,5 \%$ si lo ha hecho. Finalmente, el $51,5 \%$ ha entrenado natación entre 1 y 4 años, el $46,5 \%$ ha entrenado entre 5 y 9 años, y el $2 \%$ ha entrenado 10 años o más. Todos los deportistas y sus padres (en caso de ser necesario) firmaron un consentimiento informado para participar en la investigación.

Instrumentos: Para la presente investigación se utilizó una encuesta sociodemográfica para determinar el sexo, edad, años de actividad deportiva y presencia en la selección de Chile. Para medir los factores psicológicos de los deportistas se utilizó una adaptación del Inventario Psicológico de Ejecución Deportiva (IPED) de Hernández (2006), que consta de 42 preguntas tipo Likert, con 5 opciones que van desde 1 = casi nunca, hasta 5 = casi siempre. El IPED evalúa 7 dimensiones: autoconfianza, control de afrontamiento negativo, control atencional, control visual e imaginativo, nivel motivacional, control de afrontamiento posi- tivo y el control actitudinal; con 6 preguntas para cada dimensión. Las adaptaciones de este instrumento y sus propiedades psicométricas en nadadores chilenos se observan en la primera parte de los resultados.

Procedimiento: Los deportistas contestaron el cuestionario previo a una competencia de natación, donde fue posible reunir a todos los participantes. El tiempo aproximado de aplicación del instrumento fue de 20 minutos. Los entrenadores, los padres de los menores de edad y los deportistas firmaron un consentimiento informado para participar en la investigación.

Análisis de datos: los análisis estadísticos se realizaron con el programa SPSS 22.0 para Windows. Para conocer las propiedades psicométricas del IPED se utilizaron análisis factorial de componentes principales y rotaciones ortogonales de Varimax para cada una de las sub-escalas del cuestionario. La confiabilidad se determinó a través de análisis de alfa de Cronbach. Para conocer los niveles de desarrollo de cada sub-escala en los nadadores de Chile se utilizó estadística descriptiva, con medias y desviaciones estándar, y prueba t para comparar según sexo y entre seleccionados nacionales y no selecionados; y ANOVA para comparar entre edades. Se utilizó un nivel de confianza de 0,05 .

\section{RESULTADOS}

Análisis de las propiedades psicométricas del IPED

\section{a) Sub-escala de autoconfianza}

Se realizaron cambios semánticos en el ítem 22 de Puedo rendir hacia el rango superior de mi talento y habilidad a: Puedo rendir al máximo de mi talento y habilidad; y del ítem 36 de: Yo proyecto la imagen externa de un luchador confiado, a: Yo proyecto una imagen de un nadador confiado. Estos cambios se realizaron para evitar confusiones y contextualizarlo dentro del deporte. La sub-escala presenta un valor de KMO de 0,747 y una prueba de esfericidad de Bartlett de $\mathrm{X}^{2}=111,245 ; \mathrm{gl}=15 ; \mathrm{p}=0,000$, por 
lo que se procedió a realizar un análisis factorial de componentes principales y rotación de Varimax. Esto reveló la existencia de un componente que explica el $42,622 \%$ de la varianza (Tabla 1). Esta sub-escala obtuvo un alfa de Cronbach de 0,721.

\section{Tabla 1}

Análisis factorial de los componentes principales después de rotación Varimax de la sub-escala autoconfianza.

\begin{tabular}{lll}
\hline Ítems & Factor 1 \\
\hline $\begin{array}{l}\text { 1. } \\
\begin{array}{l}\text { Me veo más como un perdedor } \\
\text { que ganador en la competencia. }\end{array}\end{array}$ & 0,459 \\
\hline \begin{tabular}{l} 
8. Creo en mí mismo. \\
\hline $\begin{array}{l}\text { 15. Pierdo mi confianza muy } \\
\text { rápidamente. }\end{array}$
\end{tabular} & 0,736 \\
\hline $\begin{array}{l}\text { 22. Puedo rendir al máximo de mi } \\
\text { talento y habilidad. }\end{array}$ & 0,579 \\
\hline $\begin{array}{l}\text { 29. Soy un competidor fuerte } \\
\text { mentalmente. }\end{array}$ & 0,747 \\
\hline $\begin{array}{l}\text { 36. Yo proyecto la imagen externa de } \\
\text { un nadador confiado. }\end{array}$ & 0,497 \\
\hline$\%$ varianza & $\mathbf{4 2 , 6 2 2}$ \\
\hline
\end{tabular}

\section{b) Sub-escala de control de afrontamiento negativo}

Se realizaron cambios semánticos en el ítem 23 de: Mis músculos se vuelven demasiado apretados durante la competición, a: Mis músculos se aprietan demasiado durante la competición. Este cambio se realizó para evitar confusiones y contextualizarlo dentro del deporte. La subescala presenta un valor de KMO de 0,729 y una prueba de esfericidad de Bartlett de $\mathrm{X}^{2}=$ 74,$422 ; \mathrm{gl}=15 ; \mathrm{p}=0,000$, por lo que se procedió a realizar un análisis factorial de componentes principales y rotación de Varimax. Esto reveló la existencia de un componente que explica el 37,825\% de la varianza (Tabla 2). Esta sub-escala obtuvo un alfa de Cronbach de 0,650 .
Tabla 2

Análisis factorial de los componentes principales después de rotación Varimax de la sub-escala de control de afrontamiento negativo.

\begin{tabular}{|c|c|}
\hline Ítems & Factor 1 \\
\hline $\begin{array}{l}\text { 2. Me enojo y frustro durante la } \\
\text { competición. }\end{array}$ & 0,697 \\
\hline $\begin{array}{l}\text { 9. Me pongo nervioso o con miedo } \\
\text { en la competencia. }\end{array}$ & 0,641 \\
\hline $\begin{array}{l}\text { 16. Los errores me hacen sentir y } \\
\text { pensar negativamente. }\end{array}$ & 0,645 \\
\hline $\begin{array}{l}\text { 23. Mis músculos se vuelven } \\
\text { demasiado apretados durante la } \\
\text { competición. }\end{array}$ & 0,579 \\
\hline $\begin{array}{l}\text { 30. Eventos incontrolables como el } \\
\text { viento, los oponentes tramposos } \\
\text { y los malos funcionarios me son } \\
\text { muy molestos. }\end{array}$ & 0,527 \\
\hline $\begin{array}{l}\text { 37. Puedo mantener la calma durante } \\
\text { la competición. }\end{array}$ & 0,657 \\
\hline$\%$ varianza & 37,825 \\
\hline
\end{tabular}

\section{c) Sub-escala de control atencional}

Se realizaron cambios semánticos en el ítem 17 de: Puedo borrar una emoción que interfiere con rapidez y recuperar mi enfoque, a: Puedo cambiar una emoción negativa con rapidez y recuperar mi concentración; del ítem 24 de: Yo me distraigo durante la competición, a: Me distraigo durante la competición; del ítem 31 de: $M e$ encuentro a mí mismo pensando en los errores del pasado y oportunidades perdidas mientras juego, a: Suelo pensar en los errores del pasado $y$ oportunidades perdidas mientras estoy compitiendo; y el ítem 38 de: Mi concentración se rompe fácilmente, a: Pierdo la concentración fácilmente. Estos cambios se realizaron para evitar confusiones y contextualizarlo dentro del deporte. Se realizó un primer análisis factorial y prueba de confiabilidad que entrego un valor de 0,393 en el alfa de Cronbach. Se procedió a eliminar el ítem 10: Parece que mi mente empieza a correr a 100 kilómetros por hora en los momentos críticos de la competencia, con lo que aumentaron ambos parámetros psicométricos. Se realizó un segundo análisis donde la sub-escala presenta un valor de KMO de 0,731 $\mathrm{y}$ una prueba de esfericidad de Bartlett de $\mathrm{X}^{2}=$ 108,$110 ; \mathrm{gl}=10 ; \mathrm{p}=0,000$, por lo que se procedió a realizar un análisis factorial de com- 
ponentes principales y rotación de Varimax.

Esto reveló la existencia de 2 componentes que explican el $67,445 \%$ de la varianza (Tabla 3). Esta sub-escala obtuvo un alfa de Cronbach de 0,627 .

Tabla 3

Análisis factorial de los componentes principales después de rotación Varimax de la sub-escala control atencional.

\begin{tabular}{lccc}
\hline Ítems & Factor 1 & Factor 2 & \\
\hline 3. Me distraigo y pierdo la concentración durante la competición. & 0,755 & & \\
\hline $\begin{array}{l}\text { 17. Puedo cambiar una emoción negativa con rapidez y recuperar mi } \\
\text { concentración. }\end{array}$ & 0,611 & & \\
\hline 24. Me distraigo durante la competición. & 0,848 & & \\
\hline 38. Mi concentración se rompe fácilmente. & 0,833 & & \\
\hline $\begin{array}{l}\text { 31. Suelo pensar en los errores del pasado y oportunidades perdidas } \\
\text { mientras estoy compitiendo. }\end{array}$ & & 0,993 & \\
\hline \% varianza & $\mathbf{4 7 , 1 5 7}$ & $\mathbf{2 0 , 2 8 8}$ & $\mathbf{6 7 , 4 4 5}$ \\
\hline
\end{tabular}

\section{d) Sub-escala de control visuo-imaginativo}

Se realizaron cambios semánticos en el ítem 11 de: Mentalmente practico mis habilidades físicas, a: Mentalmente practico mis movimientos deportivos; del ítem 25 de: Yo me visualizo trabajando a través de situaciones difíciles antes de la competición, a: Me imagino esforzándome aún en situaciones difíciles durante la competición; y del ítem 32 de: Puedo utilizar las imágenes durante la competición y me ayudan a obtener mejores resultados, a: Puedo imaginar diversas situaciones durante la competición y me ayudan a obtener mejores resultados. Estos cambios se realizaron para evitar confusiones y contextualizarlo dentro del deporte. La subescala presenta un valor de KMO de 0,677 y una prueba de esfericidad de Bartlett de $\mathrm{X}^{2}=$ 84,$534 ; \mathrm{gl}=15 ; \mathrm{p}=0,000$ por lo que se procedió a realizar un análisis factorial de componentes principales y rotación de Varimax. Esto reveló la existencia de un componente que explica el $38,411 \%$ de la varianza (Tabla 4). Esta sub-escala obtuvo un alfa de Cronbach de 0,670 .

\section{Tabla 4}

Análisis factorial de los componentes principales después de rotación Varimax de la sub-escala control visuoimaginativo.

\begin{tabular}{lc}
\hline Ítems & Factor 1 \\
\hline 4. Antes de la competición, me imagino a mí mismo funcionando perfectamente. & 0,558 \\
\hline 11. Mentalmente practico mis movimientos deportivos. & 0,617 \\
\hline 18. Pensar en imágenes sobre mi deporte o competencia es fácil para mí. & 0,716 \\
\hline \begin{tabular}{l} 
25. Me imagino esforzándome aún en situaciones difíciles durante la competición. \\
\hline $\begin{array}{l}\text { 32. Puedo imaginar diversas situaciones durante la competición y me ayudan a obtener } \\
\text { mejores resultados. }\end{array}$
\end{tabular} & 0,506 \\
\hline 39. Cuando me visualizo a mí mismo compitiendo puedo ver y sentir las cosas claramente. & 0,751 \\
\hline \% varianza & $\mathbf{3 8 , 4 1 1}$ \\
\hline
\end{tabular}




\section{e) Sub-escala de nivel motivacional}

Se realizaron cambios semánticos en el ítem 12, de: Me mantengo trabajando duro para cumplir las metas que me he fijado a mí mismo, a: Entreno mucho para cumplir las metas que me he propuesto; del ítem 19, de: No tengo que ser empujado para jugar o practicar mucho, a: No tengo que ser obligado a entrenar mucho; y del ítem 40, de: Me despierto en la mañana y estoy muy emocionado por jugar y practicar, a: Me despierto en la mañana y estoy muy emocionado por competir y entrenar. Estos cambios se realizaron para evitar confusiones y contextualizarlo dentro del deporte. Se realizó un primer análisis factorial y prueba de confiabi- lidad que entrego un valor de 0,378 en el alfa de Cronbach. Se procedió a eliminar el ítem 19: No tengo que ser obligado a entrenar mucho, con lo que aumentaron los niveles de confiabilidad. Se realizó un segundo análisis donde la sub-escala presenta un valor de KMO de 0,698 $\mathrm{y}$ una prueba de esfericidad de Bartlett de $\mathrm{X}^{2}$ $=60,854 ; \mathrm{gl}=10 ; \mathrm{p}=0,000$, por lo que se procedió a realizar un análisis factorial de componentes principales y rotación de Varimax. Esto reveló la existencia de un componente que explica el $41,220 \%$ de la varianza (Tabla 5). Esta sub-escala obtuvo un alfa de Cronbach de 0,594 .

Tabla 5

Análisis factorial de los componentes principales después de rotación Varimax de la sub-escala nivel motivacional.

\begin{tabular}{lc}
\hline Ítems & Factor 1 \\
\hline 5. Estoy muy motivado para competir lo mejor posible. & 0,704 \\
\hline 12. Entreno mucho para cumplir las metas que me he propuesto. & 0,753 \\
\hline 26. Estoy dispuesto a dar lo que sea necesario para alcanzar todo mi potencial. & 0,780 \\
\hline 33. Me aburro y me enojo . & 0,471 \\
\hline 40. Me despierto en la mañana y estoy muy emocionado por competir y entrenar. & 0,412 \\
\hline \% varianza & $\mathbf{4 1 , 2 2 0}$ \\
\hline
\end{tabular}

\section{f) Sub-escala de control de afrontamiento positivo}

Se realizaron cambios semánticos en el ítem 6 de: Puedo mantener una fuerte emoción positiva que fluye durante la competición, a: Puedo mantener una fuerte emoción positiva durante la competición; del ítem 13, de: Yo soy capaz de disfrutar de la competición/rendimiento, incluso cuando me enfrento a muchos problemas difíciles, a: Yo soy capaz de disfrutar de la competición, incluso cuando me enfrento a muchos problemas difíciles; del ítem 20, de: Mis emociones son planas cuando las cosas se vuelven contra mí durante la competencia, a: Puedo controlar mis emociones cuando las cosas se vuelven contra mí durante la competencia; del ítem 27, de: Practico con intensidad positiva alta, a: Entreno a intensidades muy altas, dando mi mejor esfuerzo; y del ítem 41, de: Practicar mi deporte me da un verdadero sentido de alegría y satis- facción, a: Practicar mi deporte me da alegría y satisfacción. Estos cambios se realizaron para evitar confusiones y contextualizarlo dentro del deporte. Se realizó un primer análisis factorial y prueba de confiabilidad que entregó un valor de 0,309 en el alfa de Cronbach. Se procedió a eliminar el ítem 20: Puedo controlar mis emociones cuando las cosas se vuelven contra mí durante la competencia, con lo que aumentaron los niveles de ambos parámetros psicométricos. Se realizó un segundo análisis donde la sub-escala presenta un valor de KMO de 0,657 y una prueba de esfericidad de Bartlett de $\mathrm{X}^{2}=39,600 ; \mathrm{gl}=10 ; \mathrm{p}=0,000$, por lo que se procedió a realizar un análisis factorial de componentes principales y rotación de $\mathrm{Va}$ rimax. Esto reveló la existencia de 2 componentes que explican el $57,170 \%$ de la varianza (Tabla 6). Esta sub-escala obtuvo un alfa de Cronbach de 0,560. 
Tabla 6

Análisis factorial de los componentes principales después de rotación Varimax de la sub-escala de control de afrontamiento positivo.

\begin{tabular}{lccc}
\hline Ítems & Factor 1 & Factor 2 & \\
\hline $\begin{array}{l}\text { 6. Puedo mantener una fuerte emoción positiva durante la competi- } \\
\text { ción. }\end{array}$ & 0,741 & & \\
\hline $\begin{array}{l}\text { 13. Yo soy capaz de disfrutar de la competición, incluso cuando me en- } \\
\text { frento a muchos problemas difíciles. }\end{array}$ & 0,661 & & \\
\hline 34. Me desafío e inspiro en las situaciones difíciles. & 0,680 & & \\
\hline 27. Entreno a intensidades muy altas, dando mi mejor esfuerzo. & & 0,782 & \\
\hline $\begin{array}{l}\text { 41. Practicar mi deporte me da un verdadero sentido de alegría y satis- } \\
\text { facción. }\end{array}$ & 0,823 & \\
\hline \% varianza & $\mathbf{2 9 , 5 3 2}$ & $\mathbf{2 7 , 6 3 8}$ & $\mathbf{5 7 , 1 7 0}$ \\
\hline
\end{tabular}

\section{g) Sub-escala de control actitudinal}

Se realizaron cambios semánticos en el ítem 7 , de: Soy un pensador positivo durante la competición, a: Pienso positivamente durante la competición; del ítem 14, de: Mi auto-charla durante la competencia es negativa, a Hablo negativamente conmigo mismo durante la competencia; del ítem 21, de: Doy el 100\% de esfuerzo durante la práctica deportiva, a: Doy el 100\% de esfuerzo durante los entrenamientos; y del ítem 42, de: Puedo convertir la crisis en oportunidad, a: Puedo convertir los problemas en oportunidades. Estos cambios se realizaron para evi- tar confusiones y contextualizarlo dentro del deporte. La sub-escala presenta un valor de KMO de 0,766 y una prueba de esfericidad de Bartlett de $\mathrm{X}^{2}=118,834 ; \mathrm{gl}=15 ; \mathrm{p}=0,000$, por lo que se procedió a realizar un análisis factorial de componentes principales y rotación de Varimax. Esto reveló la existencia de 2 componentes que explican el $60,587 \%$ de la varianza (Tabla 7). Todos los ítems saturaron sobre 0,300 en algún factor. Esta sub-escala obtuvo un alfa de Cronbach de 0,722.

Tabla 7

Análisis factorial de los componentes principales después de rotación Varimax de la sub-escala de control de afrontamiento positivo.

\begin{tabular}{lccc}
\hline Ítems & Factor 1 & Factor 2 & \\
\hline 7. Pienso positivamente durante la competición. & 0,741 & & \\
\hline $\begin{array}{l}\text { 28. Puedo cambiar los estados de ánimo negativos en positivos mediante } \\
\text { el control de mi pensamiento. }\end{array}$ & 0,802 & & \\
\hline 42. Puedo convertir los problemas en oportunidades. & 0,844 & & \\
\hline 14. Hablo negativamente conmigo mismo durante la Competencia. & & 0,715 & \\
\hline 21. Doy el 100 por ciento de esfuerzo durante los Entrenamientos. & 0,819 & \\
\hline 35. Mis entrenadores / profesores dirían que tengo una buena actitud. & & 0,520 & \\
\hline \% varianza & $\mathbf{3 3 , 2 7 1}$ & $\mathbf{2 7 , 3 1 6}$ & $\mathbf{6 0 , 5 8 7}$ \\
\hline
\end{tabular}




\section{DISCUSIÓN}

El objeto de la presente investigación fue conocer las propiedades psicométricas del Inventario Psicológico de Ejecución Deportiva en nadadores federados de Chile. Los análisis factoriales exploratorios evidencian modelos adecuados para cada una de las sub-escalas del IPED en una muestra de nadadores de Chile. Los valores de varianza explicada oscilan entre un $37 \%$ de la sub-escala control de afrontamiento negativo, hasta un $67 \%$ de la sub-escala control atencional. Las sub-escalas de autoconfianza, control de afrontamiento negativo, control visuo-imaginativo y nivel emocional estaban compuestos por un solo factor, en tanto, las sub-escalas de control atencional, control de afrontamiento positivo y control actitudinal estaban compuestas por dos factores cada una, siendo estas tres últimas las que presentan varianzas explicadas más altas.

En la sub-escala autoconfianza, el ítem con una mayor carga factorial es: Creo en mí mismo con 0,816 , lo cual denota una característica fundamental en los deportistas, ya que suelen ser personas confiadas y con una alta autoestima (Dramisino, 2007). En la sub-escala control de afrontamiento negativo el ítem con mayor carga factorial es: Me enojo y frustro durante la competición con 0,697 , lo que podría explicarse porque la edad media de la muestra es 15,2 años, una etapa donde deportistas jóvenes son más propensos al enojo y frustración cuando las cosas no salen como planificaron (Sosa, 2005).

En la sub-escala control atencional, el ítem con la mayor carga factorial es: Suelo pensar en los errores del pasado y oportunidades perdidas mientras estoy compitiendo con 0,993 , que corresponde al único ítem del factor 2, lo que podría ser indicador de poco control del estrés y que coincide con la tendencia de la muestra a enojarse y frustrarse en contextos competitivos. En la sub-escala control visuo-imaginativo, el ítem con carga factorial más alta es: Cuando me visualizo a mí mismo compitiendo puedo ver y sentir las cosas claramente con 0,751 , lo que tiene que ver con la gran capacidad de los deportistas para enfocarse en la competencia, para pensar en imágenes sobre su deporte y para asociar emociones a la práctica deportiva (Sosa, 2005).

En la sub-escala nivel motivacional el ítem con mayor carga factorial es: Estoy dispuesto a dar lo que sea necesario para alcanzar todo mi potencial con 0,780 , dando cuenta del alto nivel de motivación intrínseca que poseen los deportistas, que los lleva a entrenar y competir hasta sus límites (Moreno, Cervelló \& González, 2007). En la sub-escala control de afrontamiento positivo el ítem con mayor carga factorial es: Practicar mi deporte me da un verdadero sentido de alegría y satisfacción con 0,823 , siendo la alegría un componente afectivo y la satisfacción un componente cognitivo del bienestar, que evalúan la calidad global de las vivencias (Veenhoven, 1994). La práctica físico-deportiva es un elemento importante que ayuda a mejorar la calidad de vida y la satisfacción por la vida (González, Huéscar \& Moreno, 2013) lo que explicaría el incremento de ambas variables con la práctica de la natación. Finalmente, en la sub-escala control actitudinal el ítem con mayor carga factorial es: Puedo convertir los problemas en oportunidades con 0,844 , lo que da cuenta de altos niveles de resiliencia de estos nadadores, que corresponde a una característica común en los deportistas de alto rendimiento (Gould, Dieffenbach \& Moffett, 2002).

La consistencia interna de cada sub-escala entrega valores de alfas de Cronbach adecuados, con dos sub-escalas con un $\alpha>0,560$; con 3 sub-escalas con $\alpha>0,600$ y 2 escalas con un $a>0,700$.

\section{CONCLUSIONES}

Los resultados de la presente investigación son una evidencia de la validez y confiabilidad del IPED para ser utilizado en nadadores de Chile, tanto en damas como varones y en edades entre 13 y 21 años.

Se recomiendan más estudios sobre las propiedades psicométricas de este instrumento en una muestra más amplia, que abarque a nadadores de todo el país. 


\section{REFERENCIAS BIBLIOGRÁFICAS}

Álvarez, O., Estevan, I., Falcó, C., HernándezMendo, A. \& Castillo, I. (2014). Perfil de habilidades psicológicas en taekwondistas universitarios y su relación con el éxito en competición. Cuadernos de Psicología del Deporte, 14(3), 13-20

Crust, L. (2009). The relationship between mental toughness and affect intensity. Personality and Individual Differences, 47(8), 959-963.

Dramisino, H. (2007). Resiliencia y deporte. Sinopsis Apsa Revista, 23(43), 23-25.

García, E., Rodríguez, M., Andrade, E. \& Arce, C. (2006). Adaptación del cuestionario MSCI para la medida de la cohesión en futbolistas jóvenes españoles. Psicothe$m a, 18(3), 668-672$

Gómez, M., Granero, A. \& Isorna, M. (2013). Análisis de los factores psicológicos que afectan a los piragüistas en el alto rendimiento, RIDEP, 35(1), 57-76.

Gould, D., Dieffenbach, K. \& Moffett, A. (2002). Psychological characteristics and their development in Olympic champions. Journal of Applied Sport Psychology, 14, 172-204.

González, G., Huéscar, E. \& Moreno, J. (2013). Satisfacción con la vida y ejercicio físico. Motricidad. European Journal of Human Movement, 30, 131-151.

Gucciardi, DF. (2012). Measuring mental toughness in sport: a psychometric examination of the psychological performance inventory-a and its predecessor. J Pers Assess, 94(4), 393-403

Hernández, A. (2006). Un cuestionario para la evaluación psicológica de la ejecución deportiva: estudio complementario entre TCT y TRI. Revista de Psicología del Deporte, 15(1), 71-93.
Hernández-Mendo, A., Morales-Sánchez, V. \& Peñalver, I. (2014). Replicación de las propiedades psicométricas del Inventario Psicológico de Ejecución Deportiva, Revista de Psicología del Deporte, 23(2), 311-324.

Izquierdo, J., Ruiz, J., Navarro, R., Mendoza, M., Rodríguez, M. \& Brito, M. (2006). Habilidades psicológicas de ejecución deportiva en jugadores de fútbol de Gran Canaria. Canarias Médica y Quirúrgica, 4(10), 39-48.

Jones, G., Hanton, S. \& Connaughton, D. (2007). A framework of mental toughness in the world's best performers. The Sport Psychologist, 21, 243-264.

Loehr, J. (1982). Athletic excellence: Mental toughness training for sports. New York: Plume Book.

Loehr, J. (1986). Mental toughness training for sports: Achieving athletic excellence. Lexington, MA: Stephen Greene Press.

Loehr, J. (1990). The Mental Game. New York: Plume Book

López, J., García, J., Berengüi, R., Martínez, A., Baños, V., Torres, M., et al. (2011). Factores físicos y psicológicos predictores del éxito en lucha olímpica. Revista de Psicología del Deporte, 20(2), 573-588.

Moreno, J., Cervelló, E. \& González, D. (2007). Analizando la motivación en el deporte: un estudio a través de la teoría de la autodeterminación. Apuntes de Psicología, 25(1), 35-51.

Orlick, T., \& Partington, J. (1988). Mental links to excellence. The Sports Psychologist, 2(2), 105-130 
Véliz, C., Maureira, F., Laurido, J.M., Carmi, N. \& Cortés, R. (2018). Propiedades psicométricas del inventario psicológico de ejecución deportiva (IPED) en una muestra de nadadores de Chile. Revista Ciencias de la Actividad Física UCM, No 19(2) julio-diciembre, 1-10. ISSN: 0719-4013.

Raimundi, M., Reigal, R. \& Mendo, A. (2016). Adaptación argentina del Inventario Psicológico de Ejecución Deportiva (IPED): validez, fiabilidad y precisión. Cuadernos de Psicología del Deporte, 16(1) 211-222

Reyes, M., Raimundi, M. \& Gómez, L. (2012). Psychological Skills Training Program in High Performance Volleyball Players. Cuadernos de Psicología del Deporte, 12(1), 9-16.

Sosa, C. (2005). Herramientas para el entrenamiento psicológico deportivo. Buenos Aires: Destino.

Veenhoven, R. (1994). El estudio de la satisfacción con la vida. Intervención Psicosocial, 3, 87-116

\section{Dirección para correspondencia}

Fernando Maureira Cid

PhD. en Educación.

Docente Escuela de Educación en Ciencias

del Movimiento y Deportes

Universidad Católica Silva Henríquez,

Santiago de Chile.

Contacto:

maureirafernando@yahoo.es

Recibido: 15/09/2017

Aceptado: 09/08/2018 